\title{
Food allergy and anaphylaxis - 2044. Component resolved allergen sensitzation profiles in shrimp allergy in the tropics
}

\author{
Irenaeus Paul Chia ${ }^{1 *}$, Gaik Chin Yap ${ }^{1}$, Bee Wah Lee ${ }^{2}$, Hugo Van Bever ${ }^{3}$, Lynette Shek Pei-Shi ${ }^{4}$, Irvin Francis A Gerez ${ }^{5}$,
} Genevieve Llanora ${ }^{5}$, Yew Kuang Cheng ${ }^{6}$, Bernard Thong ${ }^{6}$, MA Curotto De Lafaille' ${ }^{7}$ Chwee Ying Tang ${ }^{8}$

From 2nd WAO International Scientific Conference (WISC 2012)

Hyderabad, India. 6-9 December 2012

\section{Background}

Shellfish allergy is one of the commonest food allergies in the tropics.

\section{Objectives}

To evaluate the sensitization profile to a panel of allergens of dust mite(DM), shrimp and tropomyosin allergens in a cohort of shrimp allergy (SA) patients.

\section{Methods}

Serum allergen-specific IgE of 105 subjects were quantified using ImmunoCAP and ImmunoCAP ISAC biochips. The subjects were classified based on a convincing clinical history and food challenge testing (FC) (dose $=70 \mathrm{~g}$ ) to Penaeus monodon and Litopenaeus vannamei. Group1A: Either SA with FC positive to either shrimp $(\mathrm{n}=22)$ or SA admitted to emergency departments for severe reactions but no FC performed ( $n=14$ ) (total $n=36)$; and Group1B: Reported SA with FC negative $(\mathrm{n}=31)$. Group2: Shellfish tolerant DM sensitized controls $(n=38)$.

\section{Results}

All 105 subjects but one were sensitized to at least one of 3 DM tested (Dermatophagoides pteronyssinus, Dermatophagoides farina, Blomia tropicalis), with highest sensitization rates to Blo $t 5$ followed by Der $\mathrm{f} 1$. DM and shrimp tropomyosin showed high correlation $(\mathrm{p}<0.001)$. Group1A had higher rates of sensitization to tropomyosins compared to Group1B (Der p 10 [33.3\%vs9.7\%], and Pen m 1 [33.3\%vs9.7\%], $\mathrm{p}<0.037$ ); and to Group2 (Blo t 10 [19.4\% vs0\%], Pen m 1 [33.3\%vs5.3\%], Pen I 1 [27.8\%vs5.3\%], Lit v
1 [22.2\%vs5.3\%], $\mathrm{p}<0.05)$. Sensitization to Lit v 2 were higher in Group1A (22.2\%) compared to Group1B (6.5\%) and Group2 (5.2\%) ( $<<0.093)$. The sensitization rates to Lit v 3, 4 were low $(<10 \%)$. A positive test for a combination of shrimp (ImmunoCAP f24)+any tropomyosin+any shrimp allergens gave the highest sensitivity $(81.8 \%)$ to distinguish FC positive from negative subjects but had a low specificity of $24.1 \%$. The specificity was highest (93.1\%) when using a positive test for Der $\mathrm{p} 10$ or any shrimp tropomyosin, but sensitivity was low (31.8\%).

\section{Conclusions}

Tropomyosins are highly cross reactive across species and is significantly associated with SA in the tropics. ISAC Immunocap improved the accuracy to detect FC+ve SA.

\section{Author details}

${ }^{1}$ National University of Singapore, Singapore. ${ }^{2}$ The Child \& Allergy Clinic,

Singapore. ${ }^{3}$ Pediatrics, NUHS, Singapore. ${ }^{4}$ Department of Paediatrics, National University of Singapore, Singapore. ${ }^{5}$ National University Health System, University Children's Medical Institute, Department of Paediatrics, Singapore.

${ }^{6}$ Tan Tock Seng Hospital, Department of Rheumatology, Allergy and Immunology, Singapore. ${ }^{7}$ Singapore Immunology Network, Singapore. ${ }^{8}$ Rheumatology, Allergy and Immunology, Tan Tock Seng Hospital, Singapore.

Published: 23 April 2013

\section{doi:10.1186/1939-4551-6-S1-P129}

Cite this article as: Chia et al: Food allergy and anaphylaxis - 2044. Component resolved allergen sensitzation profiles in shrimp allergy in the tropics. World Allergy Organization Journal 2013 6(Suppl 1):P129.

${ }^{1}$ National University of Singapore, Singapore

Full list of author information is available at the end of the article

(c) 2013 Chia et al; licensee BioMed Central Ltd. This is an Open Access article distributed under the terms of the Creative Commons Attribution License (http://creativecommons.org/licenses/by/2.0), which permits unrestricted use, distribution, and reproduction in any medium, provided the original work is properly cited. 